\title{
Cerebral Microbleeds in Patients with Dementia with Lewy Bodies and Parkinson Disease Dementia
}

\author{
S.W. Kim, S.J. Chung, Y.-S. Oh, J.H. Yoon, M.K. Sunwoo, J.Y. Hong, J.-S. Kim, and P.H. Lee
}

\begin{abstract}
BACKGROUND AND PURPOSE: The burden of amyloid $\beta$ is greater in patients with dementia with Lewy bodies than in those with Parkinson disease dementia, and an increased amyloid $\beta$ load is closely related to a higher incidence of cerebral microbleeds. Here, we investigated the prevalence and topography of cerebral microbleeds in patients with dementia with Lewy bodies and those with Parkinson disease dementia to examine whether cerebral microbleeds are more prevalent in patients with dementia with Lewy bodies than in those with Parkinson disease dementia.
\end{abstract}

MATERIALS AND METHODS: The study population consisted of 42 patients with dementia with Lewy bodies, 88 patients with Parkinson disease dementia, and 35 controls who underwent brain MR imaging with gradient recalled-echo. Cerebral microbleeds were classified as deep, lobar, or infratentorial.

RESULTS: The frequency of cerebral microbleeds was significantly greater in patients with dementia with Lewy bodies (45.2\%) than in those with Parkinson disease dementia (26.1\%) or in healthy controls (17.1\%; $P=.017)$. Lobar cerebral microbleeds were observed more frequently in the dementia with Lewy bodies group (40.5\%) than in the Parkinson disease dementia (17\%; $P=.004)$ or healthy control (8.6\%; $P=.001)$ group, whereas the frequencies of deep and infratentorial cerebral microbleeds did not differ among the 3 groups. Logistic regression analyses revealed that, compared with the healthy control group, the dementia with Lewy bodies group was significantly associated with the presence of lobar cerebral microbleeds after adjusting for age, sex, nonlobar cerebral microbleeds, white matter hyperintensities, and other vascular risk factors (odds ratio, 4.39 [95\% Cl, 1.27-15.25]). However, compared with the healthy control group, the Parkinson disease dementia group was not significantly associated with lobar cerebral microbleeds.

CONCLUSIONS: This study showed that patients with dementia with Lewy bodies had a greater burden of cerebral microbleeds and exhibited a lobar predominance of cerebral microbleeds than did patients with Parkinson disease dementia.

ABBREVIATIONS: $\mathrm{A} \beta=$ amyloid $\beta ; \mathrm{CMB}=$ cerebral microbleed; $\mathrm{DLB}=$ dementia with Lewy bodies; PDD = Parkinson disease dementia; $\mathrm{WMH}=$ white matter hyperintensity

B oth dementia with Lewy bodies (DLB) and Parkinson disease dementia (PDD) are clinically characterized by parkinsonism and dementia but are distinctive in terms of the temporal relationship between the onset of dementia and parkinsonism. ${ }^{1,2}$ In terms of neuropathology, diffuse cortical Lewy bodies constitute the main substrate of dementia in both diseases. ${ }^{3}$ However, the

Received September 10, 2014; accepted after revision February 25, 2015.

From the Department of Neurology (S.W.K., S.J.C., P.H.L.), Yonsei University College of Medicine, Seoul, South Korea; Department of Neurology (Y.-S.O., J.-S.K.), Catholic University College of Medicine, Seoul, South Korea; Department of Neurology (J.H.Y.), Ajou University College of Medicine, Suwon, South Korea; Department of Neurology (M.K.S.), Bundang Jesaeng General Hospital, Seongnam, South Korea; Department of Neurology (J.Y.H.), Yonsei University Wonju College of Medicine, Wonju, South Korea; Severance Biomedical Science Institute (P.H.L.), Seoul, South Korea.

This research was supported by a grant of the Korea Health Technology R\&D Project through the Korea Health Industry Development Institute (KHIDI), funded by the Ministry of Health \& Welfare, Republic of Korea (grant HI14C0093). burden of amyloid $\beta(\mathrm{A} \beta)$ is greater in patients with DLB than in those with PDD. A recent neuropathologic study revealed that patients with DLB had greater plaque density than those with PDD. ${ }^{4}$ In some patients with $\mathrm{DLB}$, the density of $\mathrm{A} \beta$ deposits in the temporal lobe was comparable with that in patients with $\mathrm{Alz}$ heimer disease. ${ }^{5}$ A neuroimaging study that used ${ }^{11} \mathrm{C}$-Pittsburgh compound-B positron-emission tomography also revealed a greater $A \beta$ burden in patients with DLB than in patients with PDD. ${ }^{6}$ Thus, concomitant amyloid pathology in patients with

\footnotetext{
Please address correspondence to P.H. Lee, MD, PhD, Department of Neurology, Yonsei University College of Medicine, 250 Seongsanno, Seodaemun-gu, Seoul 120-752, South Korea; e-mail: phisland@chol.net

- Indicates open access to non-subscribers at www.ajnr.org

三 Indicates article with supplemental on-line table.

http://dx.doi.org/10.3174/ajnr.A4337
} 
DLB is considered to contribute to the early development of dementia in addition to diffuse cortical Lewy body pathology.

Cerebral microbleeds (CMBs) are small, rounded, homogeneous, hypointense lesions on $\mathrm{T} 2{ }^{\star}$-weighted gradient recalledecho imaging ${ }^{7}$ and are correlated histologically with hemosiderin deposits from the breakdown products of blood that leaks from small vessels. ${ }^{8}$ Ample evidence suggests that an increased A $\beta$ load is closely related to a higher prevalence of CMBs because the accumulation of $\mathrm{A} \beta$ within vessel walls triggers the vasculopathy of cerebral amyloid angiopathy in patients with Alzheimer disease. ${ }^{9}$ In a study of Alzheimer disease pathology, Schrag et $\mathrm{al}^{10}$ found that CMBs observed in MR imaging were correlated pathologically to microhemorrhages in areas in which $\mathrm{A} \beta$ had been deposited in adjacent vessel walls. Using ${ }^{11} \mathrm{C}$-Pittsburgh compound-B positron-emission tomography, Yates et $\mathrm{al}^{11}$ also reported that positive ${ }^{11} \mathrm{C}$-Pittsburgh compound-B positron-emission tomography scan results predicted the occurrence of lobar CMBs in healthy controls. In this study, we tested the hypothesis that a difference in $A \beta$ load between patients with DLB and those with PDD would be associated with variations in the burden and distribution pattern of CMBs in such patients. Therefore, this study was performed to explore the prevalence and topography of CMBs in patients with DLB and those with PDD.

\section{MATERIALS AND METHODS \\ Subjects}

Patient data were collected retrospectively from 3 tertiary referral hospitals (Severance Hospital and Saint Mary's Hospital [Seoul, South Korea] and Ajou University Hospital [Suwon, South Korea]). Medical and imaging data of 67 patients with DLB (43 from Severance Hospital, 13 from Ajou University Hospital, and 11 from Saint Mary's Hospital) and 132 patients with PDD (51 from Severance Hospital, 31 from Ajou University Hospital, and 50 from Saint Mary's Hospital) were reviewed. Of these patients, 42 with DLB and 88 with PDD who underwent brain MR imaging with $\mathrm{T}^{*}$-weighted gradient recalled-echo were included in the study to analyze patterns of CMBs.

DLB was diagnosed according to the revised consensus criteria for DLB, ${ }^{12}$ and PDD was diagnosed on the basis of Movement Disorder Society consensus criteria for dementia associated with Parkinson disease. ${ }^{1}$ Of 42 patients with DLB, 39 underwent ${ }^{18}$ Ffluoropropylcarbomethoxyiodophenylnortropane positron emission tomography scans, and all of them revealed decreased dopamine transporter uptake in the posterior putamen. In addition, the other 3 patients underwent cardiac ${ }^{123} \mathrm{I}$-metaiodobenzylguanidine scintigraphy, which in each of them revealed decreased uptake in the delayed image. Basic demographic data, including sex, age, and history of hypertension, diabetes mellitus, and/or cerebrovascular accident, were analyzed. A history of hypertension was defined as previous physician-diagnosed hypertension with or without prescription of antihypertensive agents, and a history of diabetes was defined on the basis of self-report or the use of hypoglycemic agents. A history of cerebrovascular accident was defined as a transient ischemic attack or stroke diagnosed by a physician. Serum levels of cholesterol measured during the diagnostic work-up were also recorded. Cognitive function was evaluated by using the Korean Mini-Mental State Examina- tion. Parkinsonian motor symptoms were assessed by using the Unified Parkinson's Disease Rating Scale Part III. Exclusion criteria included the presence of other neurodegenerative diseases that may account for dementia. Patients with a history of using drugs that cause parkinsonism (antipsychotics, gastrointestinal prokinetic drugs, antiepileptic drugs, or L-type calcium channel blockers) were excluded, and possible medical comorbidities were excluded by using laboratory tests. Patients with any history of head trauma were also excluded. Healthy age- and sex-matched elderly volunteers were included as controls for the imaging analyses. They were recruited by advertisements about the project or were healthy relatives of patients with movement disorders or dementia ( $n=35$; mean age, 73.3 years). The healthy controls did not have active neurologic disorders or cognitive complaints, and they exhibited normal performance on neuropsychological tests (minimum score of 27 on the Korean MiniMental State Examination). The University of Yonsei Institutional Review Board approved the research, which adhered to the tenets of the Declaration of Helsinki.

\section{MR Imaging Protocol}

Brain MR imaging scans were obtained by using a 3T system (Intera Achieva [Philips Healthcare, Best, the Netherlands] or Magnetom Trio [Siemens, Erlangen, Germany]). Conventional 2D $\mathrm{T} 2^{\star}$-weighted gradient recalled-echo imaging in the axial plane was obtained for the detection of CMBs with the following parameters: matrix, $100-256 \times 100-256$; section thickness, $5 \mathrm{~mm}$; repetition time, 500-1000 ms; echo time, 15-25 ms; and flip angle, $18^{\circ}-25^{\circ}$. Axial FLAIR images were also obtained to evaluate white matter hyperintensities by using the following parameters: matrix, 256-352 × 192-256; section thickness, $5 \mathrm{~mm}$; echo time, 85-138 ms; repetition time, 9600-11,000 ms; TI, 2200-2800 ms; and flip angle, $90^{\circ}$.

\section{MR Imaging Assessment}

CMBs were defined as a small, rounded, homogeneous, hypointense lesions on $\mathrm{T} 2^{\star}$-weighted gradient recalled-echo imaging; these lesions had to be distinct from other findings that mimic CMBs, and more than half of each lesion had to be surrounded by brain parenchyma. ${ }^{7}$ Other potential mimics include vessel flow voids, calcifications, and iron depositions. According to anatomic location, CMBs were classified as deep (lesions in caudate, thalamus, putamen, globus pallidus, corpus callosum, internal capsule, and external capsule), lobar (lesions in the frontal, parietal, temporal, occipital lobe, and periventricular area), or infratentorial (lesions in brain stem and cerebellum). ${ }^{13}$ The presence of CMBs in each location was assessed. White matter hyperintensities (WMHs) were defined as ill-defined hyperintense lesions on FLAIR images. WMHs were graded according to the rating scale suggested by Wahlund et al, ${ }^{14}$ which uses a 4-point scale: 0 (no lesion), 1 (focal lesion), 2 (beginning confluence), and 3 (diffuse involvement). Scoring was performed in 5 regions (frontal, parieto-occipital, temporal, infratentorial, and basal ganglia) in the right and left hemispheres; the sum of scores ranged from 0 to 30 . Brain MR imaging was reviewed independently by 2 raters, both of whom had a great deal of experience in the assessment of CMBs and the calculation of WMH scores (S.W.K. and S.J.C.). The 2 
Table 1: Demographic characteristics of the patients with DLB or PDD and healthy controls ${ }^{a}$

\begin{tabular}{|c|c|c|c|c|c|c|c|}
\hline Characteristic & Controls $(n=35)$ & Patients with DLB $(n=42)$ & Patients with PDD $(n=88)$ & $P$ & $P_{1}^{\mathrm{b}}$ & $P_{2}^{c}$ & $P_{3}{ }^{d}$ \\
\hline \multicolumn{8}{|l|}{ Sociodemographics } \\
\hline Age, y & $73.3 \pm 2.4$ & $75.1 \pm 6.1$ & $73.7 \pm 7$ & .117 & & & \\
\hline Sex, male & $10(28.6)$ & $18(42.9)$ & $32(36.4)$ & .431 & & & \\
\hline \multicolumn{8}{|l|}{ Risk factors } \\
\hline Hypertension & $17(48.6)$ & $25(59.5)$ & $41(46.6)$ & .376 & & & \\
\hline Diabetes mellitus & $7(20)$ & $16(38.1)$ & $18(20.5)$ & .071 & & & \\
\hline Cigarette smoking & $2(5.7)$ & $4(9.5)$ & $12(13.8)$ & .196 & & & \\
\hline Total cholesterol, mg/dl & $174.0 \pm 29.1$ & $160.7 \pm 30.3$ & $175.6 \pm 38.3$ & .083 & & & \\
\hline \multicolumn{8}{|l|}{ Cerebrovascular disease } \\
\hline Cortical infarction & $0(0)$ & $3(7.1)$ & $4(4.5)$ & .295 & & & \\
\hline Lacunar infarction & $7(20)$ & $14(33.3)$ & $17(19.3)$ & .184 & & & \\
\hline WMH score & $1.7 \pm 2.1$ & $5 \pm 3.7$ & $3.4 \pm 3.6$ & .001 & $<.0001$ & .082 & .121 \\
\hline K-MMSE score & $28.4 \pm 1.1$ & $19.1 \pm 5.1$ & $20.3 \pm 4.5$ & $<.0001$ & $<.0001$ & $<.0001$ & .483 \\
\hline UPDRS-III score & & $29.2 \pm 13.7$ & $28.8 \pm 15.1$ & .935 & & & \\
\hline
\end{tabular}

Note:-K-MMSE indicates Korean Mini-Mental State Examination; UPDRS-III, Unified Parkinson's Disease Rating Scale Part III.

${ }^{a}$ Values are expressed as number (\%) of subjects or mean \pm SD.

${ }^{b}$ Healthy controls vs patients with DLB.

${ }^{c}$ Healthy controls vs patients with PDD.

${ }^{d}$ Patients with DLB vs those with PDD.

raters were blind to the clinical data. Interrater reliabilities for the presence in CMBs in deep, lobar, and infratentorial areas were excellent ( $\kappa=0.86,0.93$, and 0.91 , respectively), and interrater reliability for the total WMH scores was good (correlation coefficients $=0.74)$. The presence and topography of chronic infarcts was also assessed on the basis of radiologic reports.

\section{Statistical Analysis}

Statistical analyses were performed by using SPSS software (version 18.0; IBM, Armonk, New York). Baseline demographic characteristics of the 3 groups were compared by ANOVA and $\chi^{2}$ tests for continuous and categoric variables. Post hoc comparisons with Bonferroni correction were performed to detect significant differences between each pair of groups. The $\chi^{2}$ or Fisher exact test was used to compare the number of patients with CMBs between groups. Logistic regression analyses were performed to assess the contributions of factors to the presence of lobar CMBs. Covariates considered significant at a $P$ value of $<.3$ in the univariate model were included in multivariate analysis, and a $P$ value of $<.05$ was considered statistically significant.

\section{RESULTS}

Of the 67 patients with DLB and 132 patients with PDD, 42 and 88 , respectively, were enrolled in the present study. There were no significant differences in age, sex, vascular risk factors, severity of Parkinsonian motor symptoms, or general cognition between the subjects who were and were not enrolled in the study (On-line Table). The demographic characteristics of the subjects enrolled in the study are presented in Table 1 . The groups did not differ significantly in age or sex. The frequencies of hypertension, diabetes mellitus, and smoking and the level of total cholesterol did not differ among the groups. The frequencies of both cortical and lacunar infarcts did not differ significantly among the groups. WMH scores were significantly higher in patients with DLB (5) than in healthy controls $(1.7 ; P<.0001)$ but were not significantly different from those in the PDD group (3.4; $P<.121)$. Korean Mini-Mental State Examination scores were significantly lower in the DLB (19.1) and PDD (20.3) patient groups than in the healthy
Table 2: Prevalence and distribution of cerebral microbleeds in patients with DLB or PDD and controls ${ }^{\mathrm{a}}$

\begin{tabular}{|c|c|c|c|c|c|}
\hline $\begin{array}{c}\text { Cerebral } \\
\text { Microbleed } \\
\text { Characteristic }\end{array}$ & $\begin{array}{l}\text { Healthy } \\
\text { Controls } \\
(n=35) \\
\end{array}$ & $\begin{array}{c}\text { Patients } \\
\text { with } \\
\text { DLB }(n=42)\end{array}$ & $\begin{array}{c}\text { Patients } \\
\text { with } \\
\operatorname{PDD}(n=88)\end{array}$ & $P^{\mathrm{b}} \quad P_{1}^{\mathrm{c}, \mathrm{d}} P_{2}^{\mathrm{c}, \mathrm{e}}$ & $P_{3}{ }^{c, f}$ \\
\hline Overall & $6(17.1)$ & $19(45.2)$ & $23(26.1)$ & 289. 017.009. & .029 \\
\hline Deep & $2(5.7)$ & $8(19.1)$ & $11(12.5)$ & . 216.846. & .323 \\
\hline Lobar & $3(8.6)$ & $17(40.5)$ & $15(17)$ & .001. 230. & .004 \\
\hline Infratentorial & $1(2.9)$ & $5(11.9)^{\prime}$ & $7(8)$ & .341. $212 \quad .438$ & .523 \\
\hline \multicolumn{6}{|c|}{$\begin{array}{l}\text { a Values are expressed as number (\%) of subjects. } \\
\text { b From the } \chi^{2} \text { or Fisher exact test. } \\
{ }^{c} \text { Bonferroni-corrected } P \text { values of the post hoc pair-wise comparison tests. } \\
\text { d Healthy controls versus patients with DLB. } \\
\text { e Healthy controls versus patients with PDD. } \\
\text { f Patients with DLB versus those with PDD. }\end{array}$} \\
\hline
\end{tabular}

controls $(28.4 ; P<.0001)$ but did not differ between the DLB and PDD groups.

Table 2 shows the results of $\mathrm{CMB}$ analysis among the groups. The frequency of CMBs was significantly greater in patients with DLB (45.2\%) than in those with PDD (26.1\%) and healthy controls $(17.1 \% ; P=.017$; Figure). According to post hoc analysis, the frequency of CMBs in the DLB group was significantly higher than in the PDD $(P=.029)$ and healthy control $(P=.009)$ groups, whereas the frequency of CMBs in the PDD group did not differ significantly from that in the healthy control group. The analysis of CMBs according to location revealed that the frequencies of deep and infratentorial CMBs did not differ among the healthy control (5.7\% and $2.9 \%$, respectively), PDD (12.5\% and $8 \%$, respectively), and $\mathrm{DLB}(19.1 \%$ and $11.9 \%$, respectively) groups, whereas lobar CMBs were observed more frequently in patients with DLB $(40.5 \% ; P=.001)$ than in those with PDD $(17 \%)$ and in healthy controls $(8.6 \%)$. On post hoc analysis, the frequency of lobar CMBs was higher in the DLB group than in the $\operatorname{PDD}(P=.004)$ and healthy control $(P=.001)$ groups, whereas the frequency of lobar CMBs in the PDD group did not differ significantly from that in the healthy control group.

Of the 17 patients with DLB and lobar CMBs, the CMBs were observed in the frontal areas in 5 patients $(29.4 \%)$, the parietal areas in $7(41.2 \%)$, the temporal areas in $7(41.2 \%)$, and the occipital areas in $6(35.3 \%)$. Of the 15 patients with PDD with lobar 

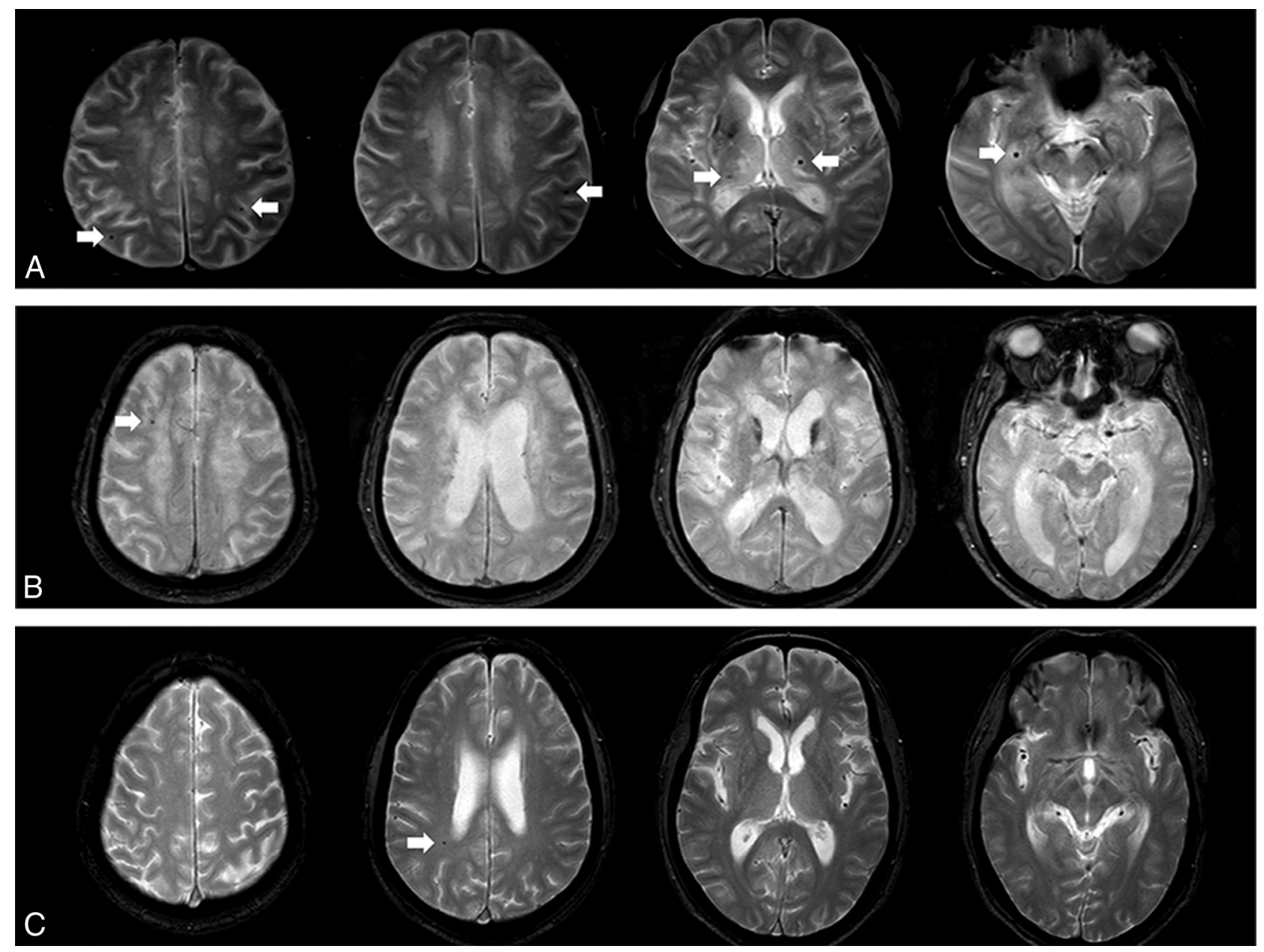

FIGURE. Examples of cerebral microbleeds observed in axial MR imaging obtained with conventional 2D T2*-weighted gradient recalled-echo. $A, M R$ imaging from a patient with DLB showing multiple CMBs (white arrows) in the bilateral thalamus, bilateral parietal lobe, and right temporal lobe. Also shown are MR images from a patient with PDD, showing a right periventricular CMB (B; white arrow), and from a healthy control, showing a right periventricular $\mathrm{CMB}(\mathrm{C}$; white arrow).

CMBs, CMBs were observed in the frontal areas in 4 patients (26.7\%), the parietal areas in 7 (46.7\%), the temporal areas in 5 $(33.3 \%)$, and the occipital areas in $2(13.3 \%)$. The topography of lobar CMBs did not differ significantly between the patients with DLB and those with PDD.

Logistic regression analyses were performed to investigate the independent determinants of lobar CMBs. When the analyses were unadjusted, DLB and WMHs were significantly associated with the presence of lobar CMBs relative to the healthy control group. After adjusting for age, sex, hypertension, diabetes mellitus, nonlobar CMBs, and WMHs, DLB was found to be significantly and independently associated with the presence of lobar CMBs relative to the control group (odds ratio, 4.39 [95\% confidence interval, 1.27-15.25]; $P=.02$ ). However, PDD was not significantly associated with lobar CMBs relative to the healthy control group (Table 3 ).

\section{DISCUSSION}

This study showed that patients with DLB had a greater burden of CMBs with lobar predominance than did patients with PDD. In addition, DLB, but not PDD, was independently associated with the presence of lobar CMBs. These data suggest that apart from
Table 3: Multivariate analysis of determinants for lobar CMBs

\begin{tabular}{|c|c|c|c|c|}
\hline \multirow[b]{2}{*}{ Variable } & \multicolumn{2}{|l|}{ Univariate } & \multicolumn{2}{|c|}{ Multivariate $^{a}$} \\
\hline & OR $(95 \% \mathrm{Cl})$ & $P$ & OR $(95 \% \mathrm{Cl})$ & $P$ \\
\hline \multicolumn{5}{|l|}{ Group } \\
\hline Controls & Reference & & Reference & \\
\hline Patients with DLB & $5.5(1.66-18.2)$ & .005 & 4.39 (1.27-15.25) & .02 \\
\hline Patients with PDD & $1.63(0.5-5.28)$ & .417 & $1.41(0.42-4.73)$ & .577 \\
\hline Hypertension & $1.79(0.85-3.78)$ & .124 & $1.85(0.52-6.6)$ & .342 \\
\hline Diabetes mellitus & $1.91(0.74-4.98)$ & .184 & $1.31(0.35-4.89)$ & .688 \\
\hline Smoking & $0.24(0.3-1.93)$ & .317 & & \\
\hline CVA & $1.33(0.33-5.41)$ & .687 & & \\
\hline Cholesterol & 0.99 (0.98-1.01) & .53 & & \\
\hline Nonlobar CMBs & $2.23(0.84-6.27)$ & .097 & $0.97(0.11-8.82)$ & .975 \\
\hline WMH & $1.31(1.12-1.53)$ & .001 & 1.44 (1.14-1.81) & .002 \\
\hline
\end{tabular}

Note:-CVA indicates cerebrovascular accident.

${ }^{a}$ On multivariate analysis, data were adjusted for age and sex.

Lewy bodies, the pathologic correlates responsible for DLB may be attributable to more frequent lobar CMBs in patients with DLB than in those with PDD.

In terms of the patterns of $\mathrm{CMB}$ distribution, hypertensive vasculopathy usually affects small perforating arterioles in the deep gray matter, thus resulting in CMBs in the basal ganglia, thalamus, and brain stem. ${ }^{8}$ In the present study, patients with 
DLB did not differ from those with PDD with respect to the CMBs located in deep or infratentorial structures, which suggests that the burden of hypertensive vasculopathy may be similar in patients with these conditions. In contrast to hypertensive vasculopathy, cerebral amyloid angiopathy usually involves leptomeningeal and cortical arterial vessels, which leads to a lobar distribution of CMBs. ${ }^{15}$ In the present study, patients with DLB exhibited a higher frequency of lobar CMBs than did healthy controls or those with PDD. Additional analysis of the topography of lobar CMBs revealed that the distribution of lobar CMBs did not differ significantly between patients with DLB and those with PDD. However, although statistical significance was not attained, lobar CMBs tended to be more frequent in the occipital areas of patients with DLB than in those with PDD. Similarly, CMBs in patients with Alzheimer disease and those with cerebral amyloid angiopathy exhibit occipital predominance. ${ }^{16,17}$ These findings suggest that there may be an association between DLB and cerebrovascular amyloid deposition.

The high prevalence and lobar predominance of CMBs in patients with DLB are consistent with the results of a previous neuropathologic study, ${ }^{18}$ in which cerebral mini-bleeds (bleeding lesions found mainly around small vessels, evident on microscopic examination but not visible macroscopically) were observed more frequently in patients with Alzheimer disease and those with DLB than in those with frontotemporal dementia and controls. Furthermore, mini-bleeds were frequently observed in the cerebral cortex and subcortical white matter of patients with DLB, and amyloid deposition in the vessels of brains with DLB was also greater than that in controls. ${ }^{18}$ Other pathologic studies also indirectly supported the relationship between DLB and CMBs. High cortical amyloid burdens and/or plaque densities were evident in patients with DLB, and these features are closely associated with CMBs. ${ }^{11}$ Ballard et $\mathrm{al}^{4}$ reported that patients with DLB had greater plaque density than those with PDD, and Deramecourt et $\mathrm{al}^{19}$ reported that patients with DLB had a considerable $\mathrm{A} \beta$ burden, comparable to that in patients with Alzheimer disease. Thus, concomitant Alzheimer disease pathology, frequently accompanied by cerebral amyloid angiopathy, may lead to an increased likelihood of CMBs in patients with DLB. On the other hand, the relationship between $\mathrm{CMBs}$ and $\mathrm{PDD}$ has not been studied in detail. Although the results of pathologic studies of cortical A $\beta$ plaque load or cerebral amyloid angiopathy pathology in patients with PDD have been inconsistent, the $\mathrm{A} \beta$ burden is regarded as less severe in patients with PDD than in those with DLB. ${ }^{4}$ Imaging studies that used ${ }^{11} \mathrm{C}$-Pittsburgh compound-B positron-emission tomography, which can detect cerebral amyloid angiopathy and diffuse plaque and neuritic plaque, showed a low amyloid burden in patients with PDD, which was comparable to those in Parkinson's disease and in healthy controls. ${ }^{20}$ Burack et $\mathrm{al}^{21}$ performed a neuropathologic examination of 3 patients with PDD who underwent ${ }^{11} \mathrm{C}$-Pittsburgh compound-B positron-emission tomography within 15 months of death, and cerebral amyloid angiopathy was absent in 2 and only mild in the third.

Another factor that may explain the higher prevalence of CMBs in patients with DLB relative to those with PDD may be differences in characteristics of the amyloid plaque. Both neu- ritic dense-core plaques and diffuse plaques are found in patients with DLB, whereas diffuse plaques are more prevalent in those with PDD. ${ }^{2,21,22}$ According to pathologic studies, compared with the diffuse plaques that are usually less harmful, dense-core plaques are known to be associated with vessel walls and to enclose vessels. ${ }^{23}$ Furthermore, a neuropathologic study in patients with Alzheimer disease and patients with Down syndrome revealed a close relationship between senile plaque and hemosiderin deposits, suggesting that dense-core plaques may be sites of microhemorrhage. ${ }^{24}$ Thus, the high proportion of dense-core plaques in patients with DLB may have contributed to the higher prevalence of CMBs in the DLB group in the present study.

The limitations of this study need to be addressed. Its retrospective design is one of the main limitations, and it is possible that selection biases affected the choice of study population. Second, we used conventional 2D T2*-weighted gradient recalled-echo, which is less sensitive than accelerated 3D $\mathrm{T} 2{ }^{\star}$-weighted gradient recalled-echo for detecting CMBs. ${ }^{25}$ Moreover, susceptibility-weighted imaging, which is more sensitive for detecting CMBs than conventional $\mathrm{T} 2{ }^{*}$-weighted gradient recalled-echo, ${ }^{26}$ has become possible. Thus, conventional 2D $\mathrm{T} 2{ }^{*}$-weighted gradient recalled-echo may have caused us to underestimate the number of CMBs. Third, the MR imaging platforms and sequence parameters used in this study were not uniform. However, the rates of CMBs in patients with DLB and in those with PDD were similar across the 3 participating hospitals $(47.4 \%, 45.5 \%$, and $41.7 \%$ in the DLB group and $26.5 \%, 28.1 \%$, and $22.7 \%$ in the PDD group at Severance Hospital, Saint Mary's Hospital, and Ajou University Hospital, respectively). Finally, other measures of $A \beta$ load (CSF A $\beta$ and positron-emission tomography) and Apolipoprotein $E$ status were not available because of the retrospective study design.

\section{CONCLUSIONS}

The present study showed that the burden and topography of CMBs differed between patients with DLB and those with PDD. Patients with DLB had a greater burden of CMBs than did patients with PDD, and they exhibited a lobar predominance of CMBs. Furthermore, DLB, but not PDD, was significantly associated with the presence of lobar CMBs.

\section{REFERENCES}

1. Emre M, Aarsland D, Brown R, et al. Clinical diagnostic criteria for dementia associated with Parkinson's disease. Mov Disord 2007;22: 1689-707; quiz 1837 Medline

2. McKeith IG, Galasko D, Kosaka K, et al. Consensus guidelines for the clinical and pathologic diagnosis of dementia with Lewy bodies (DLB): report of the consortium on DLB international workshop. Neurology 1996;47:1113-24 CrossRef Medline

3. Tsuboi Y, Dickson DW. Dementia with Lewy bodies and Parkinson's disease with dementia: are they different? Parkinsonism Relat Disord 2005;11:S47-51 CrossRef Medline

4. Ballard C, Ziabreva I, Perry R, et al. Differences in neuropathologic characteristics across the Lewy body dementia spectrum. Neurology 2006;67:1931-34 CrossRef Medline

5. Armstrong RA, Cairns NJ, Lantos PL. Beta-amyloid deposition in the temporal lobe of patients with dementia with Lewy bodies: 
comparison with non-demented cases and Alzheimer's disease. Dement Geriatr Cogn Disord 2000;11:187-92 CrossRef Medline

6. Edison P, Rowe CC, Rinne JO, et al. Amyloid load in Parkinson's disease dementia and Lewy body dementia measured with $\left[{ }^{11} \mathrm{C}\right] \mathrm{PIB}$ positron emission tomography. J Neurol Neurosurg Psychiatry 2008; 79:1331-38 CrossRef Medline

7. Greenberg SM, Vernooij MW, Cordonnier C, et al; Microbleed Study Group. Cerebral microbleeds: a guide to detection and interpretation. Lancet Neurol 2009;8:165-74 CrossRef Medline

8. Fazekas F, Kleinert R, Roob G, et al. Histopathologic analysis of foci of signal loss on gradient-echo $\mathrm{T} 2{ }^{\star}$-weighted MR images in patients with spontaneous intracerebral hemorrhage: evidence of microangiopathy-related microbleeds. AJNR Am J Neuroradiol 1999;20: 637-42 Medline

9. Goos JD, Teunissen CE, Veerhuis R, et al. Microbleeds relate to altered amyloid-[beta] metabolism in Alzheimer's disease. Neurobiol Aging 2012;33:1011.e1-9 CrossRef Medline

10. Schrag M, McAuley G, Pomakian J, et al. Correlation of hypointensities in susceptibility-weighted images to tissue histology in dementia patients with cerebral amyloid angiopathy: a postmortem MRI study. Acta Neuropathol 2010;119:291-302 CrossRef Medline

11. Yates PA, Sirisriro R, Villemagne VL, et al. Cerebral microhemorrhage and brain-amyloid in aging and Alzheimer disease. Neurology 2011;77:48-54 CrossRef Medline

12. McKeith IG, Dickson DW, Lowe J, et al; Consortium on DLB. Diagnosis and management of dementia with Lewy bodies: third report of the DLB Consortium. Neurology 2005;65:1863-72 CrossRef Medline

13. Vernooij MW, van der Lugt A, Ikram MA, et al. Prevalence and risk factors of cerebral microbleeds: the Rotterdam Scan Study. Neurology 2008;70:1208-14 CrossRef Medline

14. Wahlund LO, Barkhof F, Fazekas F, et al. A new rating scale for age-related white matter changes applicable to MRI and CT. Stroke 2001;32:1318-22 CrossRef Medline

15. Knudsen KA, Rosand J, Karluk D, et al. Clinical diagnosis of cerebral amyloid angiopathy: validation of the Boston criteria. Neurology 2001;56:537-39 CrossRef Medline
16. Pettersen JA, Sathiyamoorthy G, Gao FQ, et al. Microbleed topography, leukoaraiosis, and cognition in probable Alzheimer disease from the Sunnybrook dementia study. Arch Neurol 2008;65:790-95 CrossRef Medline

17. Rosand J, Muzikansky A, Kumar A, et al. Spatial clustering of hemorrhages in probable cerebral amyloid angiopathy. Ann Neurol 2005;58:459-62 CrossRef Medline

18. De Reuck J, Deramecourt V, Cordonnier C, et al. Prevalence of small cerebral bleeds in patients with a neurodegenerative dementia: a neuropathological study. J Neurol Sci 2011;300:63-66 CrossRef Medline

19. Deramecourt V, Bombois S, Maurage CA, et al. Biochemical staging of synucleinopathy and amyloid deposition in dementia with Lewy bodies. J Neuropathol Exp Neurol 2006;65:278-88 CrossRef Medline

20. Gomperts SN, Rentz DM, Moran E, et al. Imaging amyloid deposition in Lewy body diseases. Neurology 2008;71:903-10 CrossRef Medline

21. Burack MA, Hartlein J, Flores HP, et al. In vivo amyloid imaging in autopsy-confirmed Parkinson disease with dementia. Neurology 2010;74:77-84 CrossRef Medline

22. Harding AJ, Halliday GM. Cortical Lewy body pathology in the diagnosis of dementia. Acta Neuropathol 2001;102:355-63 Medline

23. Kumar-Singh S. Cerebral amyloid angiopathy: pathogenetic mechanisms and link to dense amyloid plaques. Genes Brain Behav 2008; 7(suppl 1):67-82 CrossRef Medline

24. Cullen KM, Kócsi Z, Stone J. Microvascular pathology in the aging human brain: evidence that senile plaques are sites of microhaemorrhages. Neurobiol Aging 2006;27:1786-96 CrossRef Medline

25. Vernooij MW, Ikram MA, Wielopolski PA, et al. Cerebral microbleeds: accelerated 3D T2* ${ }^{*}$-weighted GRE MR imaging versus conventional 2D T2* ${ }^{\star}$-weighted GRE MR imaging for detection. $R a$ diology 2008;248:272-77 CrossRef Medline

26. Nandigam RN, Viswanathan A, Delgado P, et al. MR imaging detection of cerebral microbleeds: effect of susceptibility-weighted imaging, section thickness, and field strength. AJNR Am J Neuroradiol 2009;30:338-43 CrossRef Medline 